\title{
Nanoparticles and Bacteria
}

\begin{abstract}
A concise critical review of the antibacterial features of some nanoparticles (metallic and oxide semiconductors) is presented. The review starts with a brief analysis of the physical features of nanoparticles and includes a discussion about the system nanoparticles-waterlight, which is of utmost importance in biological and medical cases. Both metal and semiconducting nanoparticles are releasing electrons under the effect of the incoming electromagnetic radiation (light). This is the source of major misunderstandings as the antibacterial features of nanoparticles can be intrinsic (in this case antibacterial capabilities will be measured in the absence of light) or mediated (in this case the antibacterial features will be observed solely under light irradiation). The critical review opens the discussion about the need for experimental measurements in dark and under the effect of light- a type of research that was typically disregarded. The main ideas are supported by examples from specialty research. New concepts as specificity and effectiveness of antibacterial features are tentatively suggested.
\end{abstract}

Keywords: Nanoparticles, Bacteria, Photochemistry, Reactive oxygen species, Electromagnetic radiation (light), Mediated antibacterial features, Effectiveness, Specificity
Volume 2 Issue 3 - 2015

\author{
Mircea Chipara,' Elamin Ibrahim, ${ }^{2}$ Brian \\ Yust,' Desiree Padilla, ${ }^{3}$ Dorina M Chipara' \\ 'Department of Physics and Astronomy, The University of Texas \\ Rio Grande Valley, USA \\ ${ }^{2}$ Department of Chemistry, The University of Texas Rio Grande \\ Valley, USA \\ ${ }^{3}$ Department of Biology, The University of Texas Rio Grande \\ Valley, USA
}

Correspondence: Mircea Chipara, Department of Physics and Astronomy, The University of Texas Rio Grande Valley, I 201 W. University Drive, Edinburg, TX 78539, USA, Tel I 9566055123 Email chippara@yahoo.com

Received: June 13,2015 | Published: September 03, 2015
Abbreviations: VB, Valence Band; CB, Conduction Band; ROS, Reactive Oxygen Species; NO, Nitric Oxide

\section{Introduction}

Antibacterial features have been reported for various nanoparticles including metallic nanoparticles (such as $\mathrm{Ag}$ and $\mathrm{Cu}$ ) and semiconducting nanoparticles. Most research was focused on semiconducting oxides (such as $\mathrm{TiO}_{2}, \mathrm{ZnO}$, and iron oxides). Reports regarding the antibacterial features of metallic and semiconducting nanoparticles are contradictory, revealing the importance of the valence state, the contribution of electromagnetic interactions, and the role of water. In such cases, it is important to disseminate between intrinsic antibacterial properties and facilitated (or mediated) antibacterial features. Intrinsic antibacterial features require a direct and major role for the nanoparticle in the antibacterial process. In the case of facilitated/mediated antibacterial properties, the presence of nanoparticles is required but not sufficient to ignite antibacterial properties. In these cases, the nanoparticles are just components of a more complex (antibacterial) mechanism, which must be activated by other parameters such as electromagnetic radiation, electrochemical processes and/or water.

Finally, the effectiveness and the specificity of the antibacterial features have not been currently investigated. The specificity should quantify the ratio between number of bacteria affected/killed by the nanoparticle and the number of healthy cells affected/killed by the same nanoparticle. Typically, the specificity applies to a pair bacteria-cell. The effectiveness of the nanoparticle as an antibacterial vector is a wide concept that comprises all pair's bacteria-cells and generates a medical landscape for the use of nanoparticles. The need for this approach derives from the fact that most studies aiming at the antibacterial features of nanoparticles are quantifying such characteristics based on the effect of nanoparticles on a culture that contains solely the bacteria and eventually some nutrients. It is easy to imagine a scenario where the nanoparticles are killing both good cells and bacteria. This aspect is generally neglected (based on the false premise that if the bacteria are killed, then medical applications are automatically implied).

\section{Infinite crystal versus finite crystal}

For a better understanding of the fine modifications caused by the transition from infinite to finite crystals, it is important to focus on the electrons. Figure 1A is represented an ideal 1-dimensional infinite crystal. In this case, the length of the crystal is infinite and the number of repeating motives $n$, is very large $(n \rightarrow \infty)$. For crystals, these repeating motives (or units) may be represented by the crystalline lattice (primitive cell for example) The electrons are moving within some potential barriers of (identical) height $V_{b}$ and thickness $b$, typically represented by the ionic moieties of the crystal. In a metal, these potential barriers are small compared to the kinetic energy of electrons. Accordingly, the electrons move almost freely within the crystal - a situation that is modeled as the free electron gas.

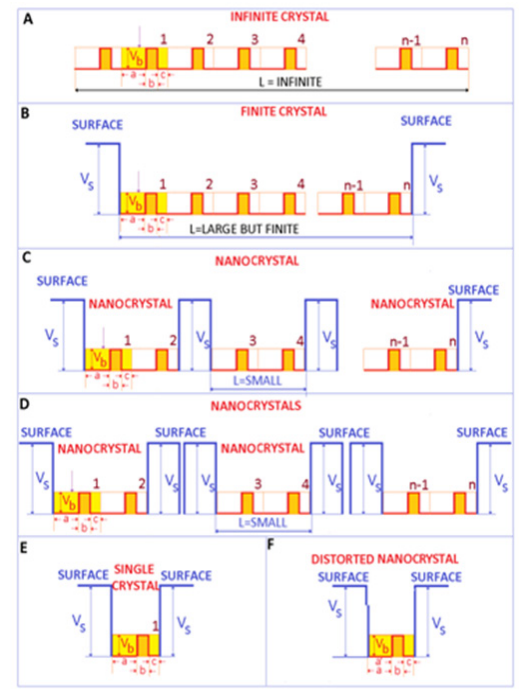

Figure I Electronic structure of a finite I dimensional crystal, showing the surface contributions, important in the photoelectric effect of metals. A. Ideal infinite crystal. B. Ideal finite crystal. C Ideal nanocrystal. D. Assembles of ideal nanocrystals. E. Single crystal. F. Distorted (real) single crystal (and nanocrystal for few repeating distorted units).

Real crystals have a finite size. Consequently, there is a surface (sometimes named boundary), which limits the extent of the crystal and separates the crystal from the environment or neighboring crystals. This implies that $\mathrm{L}$ is finite (although it can and should be very large). If $\mathrm{L}$ is very large, it is expected that surface contributions may be negligible compared to volume (bulk) contributions. 
For processes that involve the removal of an electron from the crystal (such as the photoelectric effect) as soon as the electron leaves the crystal, the crystal becomes positively charged and starts to attract the departing (negative) electron. This may provide be a qualitative representation of the potential $\mathrm{V}_{\mathrm{s}}$. Hence, in the photoelectric effect, certain energy is actually required to extract the electron from a metal. For an infinite crystal, this is shown in Figure 1A. For a finite but large (bulk) crystal this situation is shown in Figure 1B, where the number of repeating structures $\mathrm{n}$ is very large, and accordingly $\mathrm{L}$ is large although finite (for 1 dimensional metallic materials/crystals). If the size of the material (represented by L) confined between the surface potential $\mathrm{V}_{\mathrm{S}}$ is in the submicron range, a nanomaterial or nanocrystal is obtained (Figure 1C). The main difference between the bulk crystal and the nanocrystal derives from the small spatial extension of the crystal L, which should be smaller than a critical length $\xi$ - typically of the order of 100 nanometers. ${ }^{1}$ As the size of the nanocrystal L is reduced below this critical length, the energy levels of electrons are pushed up ${ }^{1-3}$ (It is important to point that the energy levels are pushed out as the size of confinement is decreased even at larger scales-not solely at the nano scale. However, this effect is stronger at shorter distances). Therefore, when dealing with bulk materials, the surface effects are typically neglected, while for nanomaterials, surface effects typically dominate over volume effects. ${ }^{2}$

Electromagnetic radiation, and in particular light may be one of the triggering parameters for the electronic processes occurring within metals and semiconductors that are finally responsible for the (chemical) attack on bacteria. The incoming light may provide the required amount of energy to promote the electron into the continuous spectrum (i.e. to free the electron). Visible light extends from about 350 to $750 \mathrm{~nm}$, which in energy units represents about 1.6 to $3.3 \mathrm{eV}$. Ultraviolet light extends from $3.3 \mathrm{eV}$ up to $10^{2} \mathrm{eV}$. The photoelectric effect theory defines the energy (work function) necessary to extract an electron from a given target. This energy can be as low as few eV for group 1, alkali metal elements $(\mathrm{Na}=2.4 \mathrm{eV}, \mathrm{K}=2.3 \mathrm{eV})$ or as high as $5 \mathrm{eV}$ for $\mathrm{C}$ (diamond). For metals such as $\mathrm{Ag}$ and $\mathrm{Cu}$, the average work function is about $4.5 \mathrm{eV}$ and $4.8 \mathrm{eV}$, respectively. Therefore, the visible light provides sufficient energy to remove electrons from finite crystals made of atoms of group 1 alkali metal elements, while the UV component may be necessary to remove electrons from other elements.

\section{Semiconductors}

A semiconducting material has a well-established electronic structure, consisting of two bands separated by an energy gap (Figure 2). In molecules with pi bonds, the molecular energy levels are split into a lower set known as Highest Occupied Molecular Orbitals or HOMO and an upper set known as Lowest Unoccupied Molecular Orbitals, or LUMO. As the number of molecules increases, the number of energy levels in both upper and lower sets increases. For sufficiently large number of molecules, HOMO degenerates into a valence band (VB) while LUMO degenerates into a conduction band (CB). ${ }^{1}$

In an ideal semiconductor, VB is typically completely filled (at low temperatures). The potential of the top of the valence band is typically labelled as $\mathrm{V}_{\mathrm{V}}$ the bottom of the conduction band is labelled as $\mathrm{V}_{\mathrm{C}}$ and the corresponding electronic states are usually completely empty at fairly low temperatures. The energy gap $\mathrm{E}_{\mathrm{G}}$ is given by $\mathrm{E}_{\mathrm{G}} \geq \mathrm{V}_{\mathrm{V}}-\mathrm{V}_{\mathrm{C}} \geq 0$. The energy gap can be as low as $0.08 \mathrm{eV}$ in alpha tin or as large as $6.36 \mathrm{eV}$ as in the case of boron nitride, which is frequently classified as a wide band semiconductor. Materials with larger energy gaps are typically classified as insulators. In the general case, the potentials
$\mathrm{V}_{\mathrm{V}}$ and $\mathrm{V}_{\mathrm{C}}$ depend on the wave vector (momentum) $\mathrm{K}$. If the maxim of the $\mathrm{V}_{\mathrm{V}}$ and the minim of the $\mathrm{V}_{\mathrm{C}}$ are occurring at the same value of $\mathrm{K}$, then the gap is direct (and the semiconductor is named direct semiconductor) with $\mathrm{E}_{\mathrm{G}}=\mathrm{V}_{\mathrm{V}}-\mathrm{V}_{\mathrm{C}} \geq 0$ (Figure 3). ${ }^{2}$ If the two extremes are not at the same wave vector then an extra energy is required to promote the electron from the valence to the conduction band. The gap is labelled as indirect gap and the semiconductor is named indirect semiconductor (Figure 3).

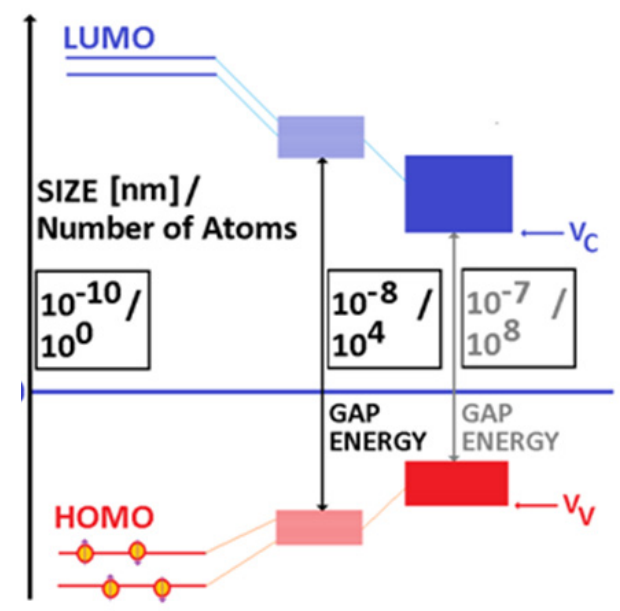

Figure 2 The formation of the energy gap as the size/ number of atoms in the particle are increased. From left to right molecules, nanometer sized semiconducting particle, bulk semicondutor particle. It is noticed that the energy gap is decreasing as the size (number of atoms) that builds up the particle is increased.

Most important direct band semiconductors are: $\mathrm{ZnO}, \mathrm{TiO}_{2}$ (rutile), $\mathrm{CuO}^{4}$ and $\mathrm{NiO}$. Among indirect band oxide semiconductors the most known is $\mathrm{TiO}_{2}$ (anatase). Some iron oxides can exhibit both direct and indirect bands. ${ }^{5,6}$ Details about the spectroscopic features of $\mathrm{TiO}_{2}$ (in dark or irradiated by electromagnetic radiation) are available in many publications. $^{7,8}$
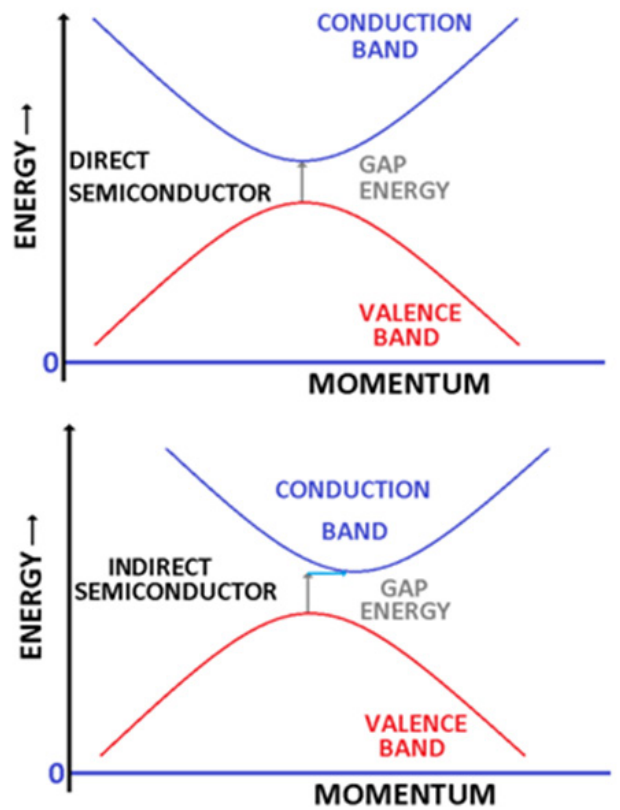

Figure 3 The energy gap in semiconductors. Upper panel depicts a direct semiconductor while the lower panel an indirect semiconductor. 
For clusters and nanoparticles, the picture is complicated by quantum and surface related issues. From the quantum point of view, the electron is localized within a potential well of a given width. As the size of the nanoparticle is decreased, the width of the potential well decreases, and the energy levels are pushed up., Experimental data indicated that actually the energy gap increases as the particle size is decreased and that the shape of nanoparticles may also affect the value of the energy gap. ${ }^{10}$ An alternative path to decrease the energy gap implies the doping of the semiconductor. For example, the doping of $\mathrm{TiO}_{2}$ by nitrogen reduced the energy gap and additionally allowed for the photoproduction of $* \mathrm{NO} .{ }^{11}$

By increasing the temperature towards room temperature, the electrons from the valence band may receive sufficient energy to be promoted into the conduction band. However, a temperature of $300 \mathrm{~K}$ corresponds to energy of about $26 \mathrm{meV}$. Typically, this energy is too small to promote the electrons from the VB into CB. Nevertheless, electromagnetic radiation and mainly visible and UV light can excite them from the valence band into the conduction band. While the electron is promoted from the valence band into the conduction band, it leaves within the valence band its image, as a positively charged quasiparticle named hole. Under the effect of an external electric field, the electrons will move towards the positive electrode while the holes will be attracted by the negative electrode.

\section{Metallic nanoparticles}

Neutral metallic nanoparticles have a reduced chemical activity and hence are expected to exhibit poor antibacterial features or even no antibacterial properties. Electrically charged metallic species (ions) exhibit chemical reactivity. The general concept is that the metallic nanoparticles that correspond to positive ions are exhibiting antibacterial properties. The generation of positive ions can be achieved either by irradiation with electromagnetic ways (typically in UV) via the photoelectric effect or electrochemically. This suggests that the antibacterial properties of metals (such as $\mathrm{Ag}, \mathrm{Au}$ ) are not active in dark, as the light is required for the formation of the positive ions. However, in the case of metallic iron, a recent paper claims antibacterial features for $\mathrm{FeO}$ (on Escherichia Coli). ${ }^{12}$ The case of nanoparticles is more complex as surface states and surface defects may result in a not negligible concentration of positive ions on a globally neutral or almost neutral nanoparticle. Unfortunately, the dissemination between dark and light-induced antibacterial features of metallic nanoparticles is not a common research objective, yet.

\section{Nanoparticles of semiconducting oxides}

Semiconducting oxides such as $\mathrm{TiO}_{2}, \mathrm{ZnO}, \mathrm{FeO}, \mathrm{Fe}_{2} \mathrm{O}_{3}, \mathrm{Fe}_{3} \mathrm{O}_{4}$, $\mathrm{NiO} . . . \mathrm{may}$ have frequently an important number of defects and in particular a large number of oxygen vacancies..$^{13}$ These can affect significantly their electronic features and may add unexpected physical properties. For example, it was speculated that hydroxyl groups may trigger magnetic features in titanium oxides. ${ }^{14}$ This demonstrates that the interaction between semiconducting oxides and water can result in complex and unexpected consequences. Poor stoichiometry should be carefully assessed, ${ }^{13}$ especially in nanomaterials where the surface to volume ratio may have an important contribution. ${ }^{13}$ The ideal nanosized semiconductor has an energy gap, whose value is controlled by size, impurities (or doping), defects, and eventually shape. The actual energy gap determines the minimum frequency of the incoming electromagnetic radiation that can excite the electron into the conduction band and eventually remove it from the semiconductor.

The analogous phenomenon in metallic nanoparticles is represented by the photoelectric effect, which finally ejects an electron from the metallic nanoparticle if the frequency of the electromagnetic radiation exceeds a certain threshold value.

\section{Water, light and semiconducting nanoparticles}

Cells, tissues and organs contain an important fraction of water. For this reason the cocktail of semiconducting or conducting nanoparticles and electromagnetic radiation is sufficient to generate free radicals or/ and ions capable to interact chemically with cells and to have sizable biological and medical consequences. Reactive oxygen species (ROS) are formed when a semiconducting (metallic) particle, and adequate electromagnetic radiation (and water) are simultaneously present. An adequate electromagnetic radiation is an electromagnetic radiation whose frequency $v$ is greater or at least equal to the ratio between the energy gap and the Planck's constant $\mathrm{h}\left(v=\mathrm{E}_{\mathrm{G}} / \mathrm{h}\right)$. Such electromagnetic radiation can promote an electron from the valence band into the conduction band leaving a hole within the valence band (Figure 4). In metals, this energy may be sufficient to extract the electron from the metallic nanoparticle. In the case of semiconductors, the energy balance schematically represented by equation (1), is responsible for the generation of electrons and holes: ${ }^{2,15}$

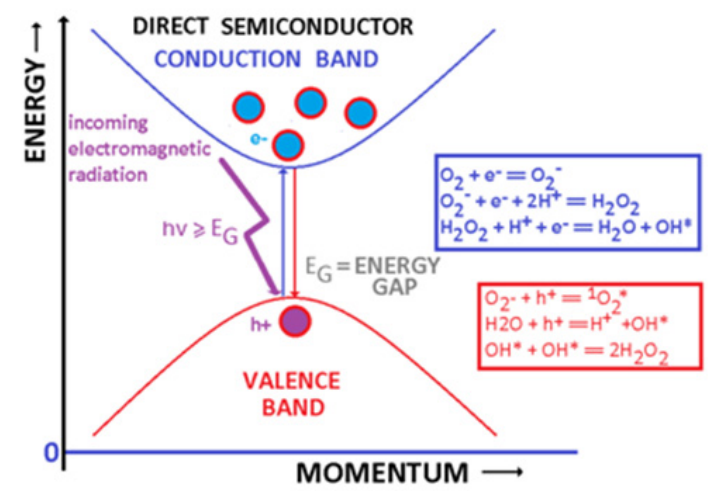

Figure 4 Water, semiconducting/metallic nanoparticles and electromagnetic radiation. A damaging cocktail in biological systems.

Semiconductor + Electromagnetic Radiation $\left(\mathbf{h v} \geq \mathbf{E}_{\mathrm{G}}\right) \rightarrow \mathbf{h}^{+}+$
e- (1) $^{-}$

Where the $\mathrm{h}^{+}$represents the hole (which is positively charged) while the $\mathrm{e}^{-}$is associated to the electron promoted into the conduction band. In most cases, ROS includes oxygen containing free radicals and ions. However, some authors include in ROS free radicals and ions that does not contain oxygen and hydrogen atoms.

Figure 4 represents some potential reactions initiated by the electron released in the conduction band and by the hole. These reactions require either the presence of water (although some reactive species like $\mathrm{O}_{2}^{-}$can be generated just in the presence of oxygen). The most important reactive oxygen species are: ${ }^{16-18}$

1) Superoxide anion $* \mathrm{O}_{2}$.

2) Hydroxide radical $* \mathrm{HO}$

3) Hydrogen peroxide $\mathrm{H}_{2} \mathrm{O}_{2}$.

4) Ozone, $\mathrm{O}_{3}$

5) Singlet oxygen

Where * identifies a free radical state. Some authors include in ROS the nitric oxide radical * $\mathrm{NO}$, hypochlorite ion $\mathrm{OCl}^{-}$, etc.... 
ROS identifies highly reactive free radicals and ions, capable of attacking biologic molecules including DNA and proteins. It is generally speculated that ROS have an important role in: ${ }^{19}$ aging, cancer, and cell evolution (birth, growth, modification, and death).

$\mathrm{TiO}_{2}$ has a complex photochemistry, analyzed in detail by. ${ }^{17,20}$ The possibility of Ti-OH structures has been confirmed by FTIR spectra. ${ }^{7}$ The presence of free radicals in $\mathrm{TiO}_{2}$ suspensions exposed to $\mathrm{H}_{2} \mathrm{O}_{2}$ and light has been confirmed by electron spin resonance spectroscopy. ${ }^{21} \mathrm{~A}$ similar mechanism for the generation of $\mathrm{Zn}^{2+}$ ions in $\mathrm{ZnO}$ exposed to $\mathrm{H}_{2} \mathrm{O}_{2}$ has been suggested for the explanation of the antibacterial features of $\mathrm{ZnO} .^{22} \mathrm{~A}$ research on nanofilms of the effect of electromagnetic radiation on $\mathrm{TiO}_{2}$ nanofilms in contact with water is described in. ${ }^{23}$ Recent studies indicated that ROS can be also generated by iron oxides $\left(\mathrm{Fe}_{3} \mathrm{O}_{4}{ }^{24}\right)$. The biological effects of ROS are discussed in several articles. ${ }^{25,26}$ The chain of chemical reactions associated with the oxidation of biological molecules is analogous to the chemical oxidative degradation of polymers, and it is frequently described by the following equations:

\section{Initiation (production of radicals)}

$\mathrm{RH}+\mathrm{O}_{2} \rightarrow \mathrm{ROOH}$ (preliminary reaction, usually $\mathrm{ROOH}$ are labile compounds).

$$
\begin{aligned}
& \mathrm{ROOH} \rightarrow * \mathrm{RO}+* \mathrm{HO} \\
& \mathrm{ROOH}+* \mathrm{OH} \rightarrow * \mathrm{ROO}+\mathrm{H}_{2} \mathrm{O} \\
& \mathrm{RH}+* \mathrm{OH} \rightarrow * \mathrm{R}+\mathrm{H}_{2} \mathrm{O}
\end{aligned}
$$

\section{Propagation}

$$
* \mathrm{R}+\mathrm{O}_{2} \rightarrow * \mathrm{ROO}
$$

\section{Transfer reactions}

$$
\begin{aligned}
& * \mathrm{ROO}+\mathrm{RH} \rightarrow * \mathrm{R}+\mathrm{ROOH} \\
& * \mathrm{RO}+\mathrm{RH} \rightarrow * \mathrm{R}+\mathrm{ROH}
\end{aligned}
$$

\section{Termination Reactions}

$$
\begin{aligned}
& * \mathrm{R}+* \mathrm{R} \rightarrow \mathrm{R}-\mathrm{R} \\
& * \mathrm{RO}_{2}+* \mathrm{R} \rightarrow \mathrm{R}-\mathrm{RO}_{2} \\
& * \mathrm{RO}_{2}+* \mathrm{RO}_{2} \rightarrow 2 \mathrm{RO}_{2}
\end{aligned}
$$

Consequently, the antibacterial properties may be in some cases a result of the simultaneous presence of 3 factors, oxide semiconducting (or metallic) particles, electromagnetic radiation (of adequate wavelength), and water. In the case of $\mathrm{TiO}_{2}$ nanotubes the outcome of this cocktail is an obvious antibacterial behavior, as confirmed in. ${ }^{27}$ This manuscript supports the observation that actually the antibacterial features may not be a direct result of the presence of $\mathrm{TiO}_{2}$ but a consequence of ROS interacting with the membrane of bacteria. A better knowledge of the interactions between $\mathrm{TiO}_{2}$ (or $\mathrm{ZnO}$ ), electromagnetic radiation, and water is mandatory in understanding the chemical processes and risks implied by the use of sunscreens and/or cosmetics based on oxide semiconductors. ${ }^{28}$ Recent studies suggest that sonication can replace the electromagnetic radiation in the generation of ROS in $\mathrm{TiO}_{2} \cdot{ }^{17,29}$

\section{Potential biological and medical applications}

Metallic nanoparticles such as $\mathrm{Ag}$, and $\mathrm{Au}^{30}$ have been known to have beneficial medical applications since ancient Greece. Recent studies indicated that $\mathrm{Au}$ and $\mathrm{Ag}$ and in particular $\mathrm{Ag}$ ions have antifungal properties..$^{31}$ Unfortunately, the effect of light on the antifugal features has not been investigated. Spectroscopic investigations revealed the formation of a biocomplex between human hemoglobin and silver nanoparticles. ${ }^{32}$ Later, it was recognized that actually silver and gold have important antibacterial features against both Gramm positive and Gramm negative bacteria, and that these features are enhanced if the particles are well disperse and in colloidal form. ${ }^{30}$ It was concluded that silver has the best antimicrobial features. Unfortunately, the authors did not disseminate between light and dark antibacterial features and did not try to monitor the eventual production and evolution of ROS species. Gold nanoparticles deposited on zeolites have efficient antibacterial features (Escherichia coli and Salmonella typhi). Unfortunately, dark tests have not been reported. ${ }^{33}$

It was reported $\mathrm{d}^{22,34}$ that nanoparticles of semiconducting oxides are more efficient as antibacterial agents against Gramm positive the Gramm negative strains of bacteria, with stronger antibacterial activities for $\mathrm{ZnO}$ (compared to $\mathrm{CuO}$ and $\mathrm{Fe}_{2} \mathrm{O}_{3}$ ). The authors speculated that the surface to volume ratio has a sizable contribution on the antibacterial features. ${ }^{34}$ The study was performed on a system that included nutrients, various bacteria strains, and nanoparticles. The presence/absence of light was not discussed. The effect of nanoparticles' concentration was not investigated. Nice FTIR and WAXS data on nanoparticles are reported.

While not directly connected to medical applications, ${ }^{35}$ focuses on the synthesis and photocatalitic activity of $\mathrm{TiO}_{2}$, describing the chemical reactions occurring in $\mathrm{TiO}_{2}$ under the effect of electromagnetic radiation (solar radiation). This is a good starting point in the analysis of the use of semiconducting oxides $\left(\mathrm{TiO}_{2}\right.$ and $\mathrm{ZnO}$ ) as major components of sunscreens. In order to understand the role of $\mathrm{TiO}_{2}$ nanoparticles in biological system we have to start from $\mathrm{TiO}_{2}$ nanoparticles and water. This is exactly the subject of the research reported in. ${ }^{36}$

The production of ROS by semiconducting/conducting nanoparticle (such as $\mathrm{TiO}_{2}$ ) and light in cell cultures (human cervical carcinoma cells $\mathrm{HeLa}$ ) has been confirmed. ${ }^{11}$ A study aiming directly at $\mathrm{TiO}_{2}$ exposed to $\mathrm{H}_{2} \mathrm{O}_{2}$ in ambient light and dark demonstrated that free oxygen containing radicals (ROS) could be generated in the absence of light if $\mathrm{H}_{2} \mathrm{O}_{2}$ is present. The formation of hydroperoxyl and hydroxyl free radicals was confirmed by electron spin resonance spectroscopy.

An excellent example about the complex biological consequences of the cocktail water, semiconducting nanoparticles, and light is provided by the demonstration that $\mathrm{TiO}_{2}$ nanoparticles are phototoxic to marine phytoplankton. ${ }^{37}$ This remarkable study includes $\mathrm{TiO}_{2}$ nanoparticles, water, and electromagnetic radiation. It is revealed that $\mathrm{UV}$ radiation is required to turn on the antibacterial capabilities of $\mathrm{TiO}_{2}$.

Nanoparticles of $\mathrm{TiO}_{2}$ are frequently used in cosmetics either as white pigment or for UV protection (sunscreen). ${ }^{7,38}$. Typically, the UV domain is divided into UVB ranging from 290 to $320 \mathrm{~nm}$, UVA-2 between 320 and $340 \mathrm{~nm}$, and UVA-1 between 340 and 400 $\mathrm{nm}$. The large absorbance of $\mathrm{TiO}_{2}$ in UV is widely recognized and documented. Micron sized $\mathrm{TiO}_{2}$ particles are efficient in blocking UVB type of radiation while micron sized $\mathrm{ZnO}$ nanoparticles are preferentially absorbing in UVA-1 domain. ${ }^{28}$ Nevertheless, as the UV radiation is absorbed, electron-hole pairs are generated and ROS production is ignited in both $\mathrm{ZnO}$ and $\mathrm{TiO}_{2}$ particles. The decrease of the size of $\mathrm{TiO}_{2}$ and $\mathrm{ZnO}$ particles into the nanometer range increases the energy gap but this modification is not sufficiently large to make the production of pair electron-hole under the effect of the daily light 
negligible. Detailed investigations on the antibacterial features of $\mathrm{ZnO} / \mathrm{SnO}_{2}$ composite films revealed that the antibacterial activity (for Escherichia coli) is enhanced by UV irradiation but it is not completely stopped in dark. ${ }^{18}$ The fact that some films retained even in the dark some antibacterial activity may indicate the presence of trapped oxygen species (assuming that a perfect dark was achieved) and may provide a path to intrinsic, passive antibacterial features (not mediated by light). A study on antibacterial features of some metal oxides $(\mathrm{ZnO}$, $\mathrm{CuO}$, and $\mathrm{Fe}_{2} \mathrm{O}_{3}$ ) nanoparticle revealed some antibacterial activity in all of the studied oxides, with $\mathrm{ZnO}$ showing the strongest antibacterial activity and $\mathrm{Fe}_{2} \mathrm{O}_{3}$ exhibiting the weakest antibacterial features. It was concluded that these oxides are more efficient against Gramm positive than Gramm negative strains, and that the surface appears to enhance the antibacterial features. ${ }^{34}$ No detail about the presence of light during this experiment was provided. Antibacterial features have also been reported in several semiconductors (not necessarily oxide semiconductors) such as CdS. ${ }^{39}$

\section{Conclusion}

Metallic and semiconducting particles and nanoparticles may exhibit antibacterial features, especially if positively charged. The electromagnetic radiation may trigger the generation of ROS under the presence of light. ${ }^{40}$ This explains the reason for which some studies confirmed the antibacterial features of these particles/ nanoparticles while other researchers failed to confirm these results. Sonication may eventually replace the electromagnetic radiation in the generation of ROS in oxide semiconductors (or metals exposed to oxygen atmosphere). Aiming towards medical oriented results, it is important to conduct the experiments either under light illumination (for tissues and organs that can be easily illuminated such as the skin) or under total dark (for some internal organs). The direct production of ROS by particles or nanoparticles is not impossible. Such a situation will describe a ROS intrinsic system. However, in most cases light is required. In this case, the particle/nanoparticle plays an important role in the ROS production but is not efficient to ignite ROS generation.

ROS is a double sword. It is a collection of chemically active species (free radicals, ions, and ion-free radicals) capable of attacking and destroying biological cells at the molecular scale. From this angle, ROS is an efficient protection mechanism. However, ROS is also able to attack healthy cells, becoming dangerous to them. This process should be handled with care and activated only under certain circumstances. Therefore, a reliable procedure to turn on and off ROS can become instrumental in the protection of cells.

The cocktail of $\mathrm{TiO}_{2}$ nanoparticles and UV irradiation was used to generate ROS species in various cells, in a tentative to kill cancer cells. Preliminary tests in the laboratory demonstrated that the procedure is efficient and several improvements have already been suggested..$^{11}$ Nevertheless, such an approach can be used solely for few cancers, where the activation by visible of UV light is possible. Endoscopic illumination - although possible will not convert the use of this cocktail into a treatment option for any kind of cancer but may add an important medical tool. As both Visible and UV radiation has a thin penetration depth within the human body, an important fraction of these studies are mostly theoretical.

Future research will need to disseminate between zero valence states and neutral states, to quantify accurately the effect of electromagnetic radiation and water, and in certain occasion to consider the potential effects of the oxygen presence. At least, the problem of nanoparticles (or colloids) has to be better addressed, with emphasize on surface states-where trapped positive ions can be the source of some existing discrepancies regarding antibacterial features.
Specificity and efficiency remain ultimate goals before medical/human applications. ROS should be use with care as it is also considered as a major responsible for cancer (at chemical and molecular level) ${ }^{41}$

\section{Acknowledgments}

None.s

\section{Conflicts of interest}

None.

\section{References}

1. Smith AM, Nie S. Semiconductor Nanocrystals: Structure, Properties, and Band Gap Engineering. Acc Chem Res. 2010;43(2):190-200.

2. Brus LE, Trautman JK. Nanocrystals and nano-optics. Philos Trans Phys Sci Eng. 2008;353(1703):313-321.

3. Lin $\mathrm{KF}$, Cheng HM, Hsu HC, et al. Band gap variation of sizecontrolled $\mathrm{ZnO}$ quantum dots synthesized by sol-gel method. Chem Phys Lett. 2005;409(4-6):208-211.

4. Kidowaki H, Oku T, Akiyama T. Fabrication and characterization of $\mathrm{CuO} / \mathrm{ZnO}$ solar cells. J Phys Conf Ser. 2012;352(1):012022.

5. Mallick P, Dash BN. X-ray Diffraction and UV-Visible Characterizations of $\alpha-\mathrm{Fe}_{2} \mathrm{O}_{3}$ Nanoparticles Annealed at Different Temperature. Scientific \& Academic Publishing. 2013;3(5):130-134.

6. Al-Kuhaili MF, Saleem M, Durrani SMA. Optical properties of iron oxide $\left(\alpha-\mathrm{Fe}_{2} \mathrm{O}_{3}\right)$ thin films deposited by the reactive evaporation of iron. J Alloys Compd. 2012;521(25):178-182.

7. Gao Y, Masuda Y, Seo WS, et al. $\mathrm{TiO}_{2}$ nanoparticles prepared using an aqueous peroxotitanate solution. Ceram Int. 2004;30(7):1365-1368.

8. Kumar PM, Badrinarayanan S, Sastry M. Nanocrystalline $\mathrm{TiO}_{2}$ studied by optical, FTIR and X-ray photoelectron spectroscopy: correlation to presence of surface states. Thin Solid Films. 2000;358(1-2):122-130.

9. Vahala KJ. Quantum Box Fabrication Tolerance and Size Limits in Semiconductors and Their Effect on Optical Gain. IEEE J Quantum Electron. 1988;24(3):523-530.

10. Wang Y, Ouyang G, Wang LL, et al. Size- and compositioninduced band-gap change of nanostructured compound of II-VI semiconductors. Chem Phys Lett. 2008;463(4-6):383-386.

11. Li Z, Pan X, Wang T, et al. Comparison of the killing effects between nitrogen-doped and pure $\mathrm{TiO}_{2}$ on HeLa cells with visible light irradiation. Nanoscale Res Lett. 2013;8(1):96.

12. Lee C, Yeon KJ, Lee WI, et al. Bactericidal Effect of Zero-Valent Iron Nanoparticles on Escherichia Coli. Environ Sci Technol. 2008;42(13):4927-4933.

13. Grujić-Brojčin M, Šćepanović M, Dohčević-Mitrović Z, et al. Infrared study of nonstoichiometric anatase $\mathrm{TiO}_{2}$ nanopowders. Sci Sinter. 2006;38(2):183.

14. Skomski R, Wei X, Balamurugan B, et al. Hydroxyl-Induced Magnetism in Ti Oxides. IEEE Trans. 2010;46(6):2427-2430.

15. Lauer JL, Shohet JL, Hansen RW. Measuring vacuum ultraviolet radiation-induced damage. J Vac Sci Technol A. 2003;21(4):1253-1259.

16. Jeong YS, Oh WK, Kim S, et al. Cellular uptake, cytotoxicity, and ROS generation with silica/conducting polymer core/shell nanospheres. Biomaterials. 2011;32(29):7217-7225.

17. Guo Y, Cheng C, Wang J, et al. Detection of reactive oxygen species (ROS) generated by $\mathrm{TiO}_{2}(\mathrm{R}), \mathrm{TiO}_{2}(\mathrm{R} / \mathrm{A})$ and $\mathrm{TiO}_{2}(\mathrm{~A})$ under ultrasonic and solar light irradiation and application in degradation of organic dyes. J Hazard Mater. 2011;192(2):786-793. 
18. Talebian N, Nilforoushan MR, Zargar EB. Enhanced antibacterial performance of hybrid semiconductor nanomaterials: $\mathrm{ZnO} /$ $\mathrm{SnO}_{2}$ nanocomposite thin films. Appl Surf Sci. 2011;258(1):547-555.

19. Ray PD, Huang BW, Tsuji Y. Reactive oxygen species (ROS) homeostasus and redox regulation in cellular signaling. Cell Sugnal. 2012;24(5):981-990.

20. Yates JT. Photochemistry on $\mathrm{TiO}_{2}$ : Mechanisms behind the surface chemistry. Surf Sci. 2009;603(10-12):1605-1612.

21. Sánchez LD, Taxt-Lamolle SFM, Hole EO, et al. $\mathrm{TiO}_{2}$ suspension exposed to $\mathrm{H}_{2} \mathrm{O}_{2}$ in ambient light or darkness: Degradation of methylene blue and EPR evidence for radical oxygen species. Appl Catal B Environ. 2013;142-143:662-667.

22. Fakhroueian Z, Harsini FM, Chalabian F, et al. Influence of Modified ZnO Quantum Dots and Nanostructures as New Antibacterials. Adv Nanoparticles. 2013;2(3):247-258.

23. Nakamura R, Imanishi A, Murakoshi K, et al. In situ FTIR studies of primary intermediates of photocatalytic reactions on nanocrystalline $\mathrm{TiO}_{2}$ films in contact with aqueous solutions. J Am Chem Soc. 2003;125(24):7443-7450.

24. Tran N, Mir A, Mallik D, et al. Bactericidal effect of iron oxide nanoparticles on Staphylococcus aureus. Int J Nanomedicine. 2010;5:277-283.

25. Le Bras M, Clément MV, Pervaiz S, et al. Reactive oxygen species and the mitochondrial signaling pathway of cell death. Histol Histopathol. 2005;20(1):205-219.

26. Cai R, Kubota Y, Shuin T, et al. Induction of Cytotoxicity by Photoexcited $\mathrm{TiO}_{2}$ Particles. Cancer Res. 1992;52(8):2346-2348.

27. Podporska-Carroll J, Panaitescu E, Quilty B, et al. Antimicrobial properties of highly efficient photocatalytic $\mathrm{TiO}_{2}$ nanotubes. Appl Catal B Environ. 2015;176-177:70-75.

28. Smijs TG, Pavel S. Titanium dioxide and zinc oxide nanoparticles in sunscreens: Focus on their safety and effectiveness. Nanotechnol Sci Appl. 2011;4:95-112.

29. Wang J, Guo Y, Liu B, et al. Detection and analysis of reactive oxygen species (ROS) generated by nano-sized $\mathrm{TiO}_{2}$ powder under ultrasonic irradiation and application in sonocatalytic degradation of organic dyes. Ultrason Sonochem. 2011;18(1):177-183.
30. Zhou Y, Kong Y, Kundu S, et al. Antibacterial activities of gold and silver nanoparticles against Escherichia coli and bacillus CalmetteGuerin. J Nanobiotechnology. 2012;10:19.

31. Jo YK, Kim BH, Jung G. Antifungal Activity of Silver Ions and Nanoparticles on Phytopathogenic Fungi. Plant Dis. 2009;93(10):1037-1043.

32. Mahato M, Pal P, Tah B, et al. Study of silver nanoparticlehemoglobin interaction and composite formation. Colloids Surfaces B Biointerfaces. 2011;88(1):141-149.

33. Lima E, Guerra R, Lara V, et al. Gold nanoparticles as efficient antimicrobial agents for Escherichia coli and Salmonella typhi. Chem Cent J. 2013;7(1):11.

34. Azam A, Ahmed AS, Oves M, et al. Antimicrobial activity of metal oxide nanoparticles against Gram-positive and Gram-negative bacteria: A comparative study. Int J Nanomedicine. 2012;7:6003-6009.

35. Ba-Abbad MM, Kadhum AAH, Mohamad AB, et al. Synthesis and catalytic activity of TiO 2 nanoparticles for photochemical oxidation of concentrated chlorophenols under direct solar radiation. Int $J$ Electrochem Sci. 2012;7(2012):4871-4888.

36. Bezrodna T, Gavrilko T, Puchkovska G, et al. Spectroscopic study of $\mathrm{TiO}_{2}$ (rutile)-benzophenone heterogeneous systems. J Mol Struct. 2002;614(1-3):315-324

37. Miller RJ, Bennett S, Keller A a, et al. $\mathrm{TiO}_{2}$ nanoparticles are phototoxic to marine phytoplankton. PLoS One. 2012;7(1):e30321.

38. Schalka S, Reis VMS Dos. Sun protection factor: meaning and controversies. An Bras Dermatol. 2011;86(3):507-515.

39. Jing Z, Tan L, Li F, Wang J. Photocatalytic and antibacterial activities of CdS nanoparticles prepared by solvothermal method. IJC-A. 2013;52(1):57-62.

40. Geiseler B, Miljevic M, Müller P, et al. Phototriggered production of reactive oxygen species by $\mathrm{TIO}_{2}$ nanospheres and rods. J Nanomater. 2012:9.

41. Waris G, Ahsan H. Reactive oxygen species: role in the development of cancer and various chronic conditions. J Carcinog. 2006;5:14. 